\title{
THE EXACT ASYMPTOTIC BEHAVIOUR OF THE UNIQUE SOLUTION TO A SINGULAR DIRICHLET PROBLEM
}

\author{
ZHIJUN ZHANG AND JIANNING YU
}

Received 23 August 2005; Revised 10 November 2005; Accepted 13 November 2005

By Karamata regular variation theory, we show the existence and exact asymptotic behaviour of the unique classical solution $u \in C^{2+\alpha}(\Omega) \cap C(\bar{\Omega})$ near the boundary to a singular Dirichlet problem $-\Delta u=g(u)-k(x), u>0, x \in \Omega,\left.u\right|_{\partial \Omega}=0$, where $\Omega$ is a bounded domain with smooth boundary in $\mathbb{R}^{N}, g \in C^{1}((0, \infty),(0, \infty)), \lim _{t \rightarrow 0^{+}}(g(\xi t) / g(t))=\xi^{-\gamma}$, for each $\xi>0$ and some $\gamma>1$; and $k \in C_{\text {loc }}^{\alpha}(\Omega)$ for some $\alpha \in(0,1)$, which is nonnegative on $\Omega$ and may be unbounded or singular on the boundary.

Copyright (c) 2006 Z. Zhang and J. Yu. This is an open access article distributed under the Creative Commons Attribution License, which permits unrestricted use, distribution, and reproduction in any medium, provided the original work is properly cited.

\section{Introduction and the main results}

The purpose of this paper is to investigate the existence and exact asymptotic behaviour of the unique classical solution near the boundary to the following model problem:

$$
-\triangle u=g(u)-k(x), \quad u>0, x \in \Omega,\left.u\right|_{\partial \Omega}=0
$$

where $\Omega$ is a bounded domain with smooth boundary in $\mathbb{R}^{N}(N \geq 1), k \in C_{\text {loc }}^{\alpha}(\Omega)$ for some $\alpha \in(0,1)$, which is nonnegative on $\Omega$, and $g$ satisfies

$\left(g_{1}\right) g \in C^{1}((0, \infty),(0, \infty)), g^{\prime}(s) \leq 0$ for all $s>0, \lim _{s \rightarrow 0^{+}} g(s)=+\infty$.

The problem arises in the study of non-Newtonian fluids, boundary layer phenomena for viscous fluids, chemical heterogeneous catalysts, as well as in the theory of heat conduction in electrical conductive materials (see $[4,7,12,14]$ ).

The main feature of this paper is the presence of the two terms, the singular term $g(u)$ which is regular varying at zero of index $-\gamma$ with $\gamma>1$ and includes a large class of singular functions, and the nonhomogeneous term $k(x)$, which may be singular on the boundary.

This type of nonlinear terms arises in the papers of Díaz and Letelier [6], Lasry and Lions [10] for boundary blow-up elliptic problems. 
For $k \equiv 0$ on $\Omega$, problem (1.1) is the following one:

$$
-\Delta u=g(u), \quad u>0, x \in \Omega,\left.u\right|_{\partial \Omega}=0 .
$$

The problem was discussed and extended to the more general problems in a number of works, see, for instance, [4, 5, 7, 8, 11, 14-17]. Fulks and Maybee [7], Stuart [14], Crandall et al. [4] showed that if $g$ satisfies $\left(g_{1}\right)$, then problem (1.2) has a unique solution $u_{0} \in C^{2+\alpha}(\Omega) \cap C(\bar{\Omega})$. Moreover, Crandall et al. [4, Theorems 2.2 and 2.7] showed that there exist positive constants $C_{1}$ and $C_{2}$ such that

(I) $C_{1} \psi(d(x)) \leq u_{0}(x) \leq C_{2} \psi(d(x))$ near $\partial \Omega$, where $d(x)=\operatorname{dist}(x, \partial \Omega)$, $\psi \in C[0, a] \cap C^{2}(0, a]$ is the local solution to the problem

$$
-\psi^{\prime \prime}(s)=g(\psi(s)), \quad \psi(s)>0, \quad 0<s<a, \quad \psi(0)=0 .
$$

Then, for $g(u)=u^{-\gamma}, \gamma>0$, Lazer and McKenna [11], by construction of the global subsolution and supersolution, showed that $u_{0}$ has the following properties:

$\left(\mathrm{I}_{1}\right)$ if $\gamma>1$, then $C_{1}\left[\phi_{1}(x)\right]^{2 /(1+\gamma)} \leq u_{0}(x) \leq C_{2}\left[\phi_{1}(x)\right]^{2 /(1+\gamma)}$ on $\bar{\Omega}$;

( $\left.\mathrm{I}_{2}\right)$ if $\gamma>1$, then $u_{0} \notin C^{1}(\bar{\Omega})$;

$\left(\mathrm{I}_{3}\right) u_{0} \in H_{0}^{1}(\Omega)$ if and only if $\gamma<3$, this is a basic character to problem (1.2) in the case,

where $\phi_{1}$ is the eigenfunction corresponding to the first eigenvalue of problem $-\Delta u=\lambda u$ in $\Omega$, and $\left.u\right|_{\partial \Omega}=0$.

Most recently, when $\int_{1}^{\infty} g(s) d s<\infty$, in [16], we showed that

(II) $C_{1} \psi(d(x)) \leq u_{0}(x) \leq C_{2} \psi(d(x))$, on $\bar{\Omega}$,

where $\psi \in C[0, \infty) \cap C^{2}(0, \infty)$ is the unique global solution to the problem

$$
-\psi^{\prime \prime}(s)=g(\psi(s)), \quad \psi(s)>0, \quad s>0, \quad \psi(0)=0, \quad \lim _{s \rightarrow \infty} \psi(s)=\beta \geq 0 .
$$

Moreover, assume $g$ satisfies $\left(g_{1}\right)$ and

$\left(\mathrm{g}_{2}\right)$ there exist positive constants $C_{0}, \eta_{0}$ and $\gamma \in(0,1)$ such that $g(s) \leq C_{0} s^{-\gamma}$, for all $s \in\left(0, \eta_{0}\right)$

$\left(g_{3}\right)$ there exist $\theta>0$ and $t_{0} \geq 1$ such that $g(\xi t) \geq \xi^{-\theta} g(t)$ for all $\xi \in(0,1)$ and $0<t \leq$ $t_{0} \xi$

$\left(\mathrm{g}_{4}\right)$ the mapping $\xi \in(0, \infty) \rightarrow T(\xi)=\lim _{t \rightarrow 0^{+}}(g(\xi t) / \xi g(t))$ is a continuous function. Ghergu and Rădulescu [8] showed that problem (1.2) has a unique solution $u_{0} \in C^{1,1-\alpha}(\bar{\Omega})$ $\cap C^{2}(\Omega)$ satisfying

$$
\lim _{d(x) \rightarrow 0} \frac{u_{0}(x)}{\psi(d(x))}=\xi_{0}
$$

where $T\left(\xi_{0}\right)=1$, and $\psi \in C^{1}[0, a] \cap C^{2}(0, a]\left(a \in\left(0, \eta_{0}\right)\right)$ is the local solution to problem (1.3).

For $k \leq 0$ on $\Omega, k \in L^{p}(\Omega)$ with $p>N / 2$, and $g(u)=u^{-\gamma}, \gamma>0$, Aranda and Godoy [1] showed that problem (1.1) has a unique solution $u \in W_{\text {loc }}^{2, p}(\Omega) \cap C(\bar{\Omega})$.

Most recently, applying Karamata regular variation theory, Cîrstea and Rădulescu [3] and Cîrstea and Du [2] studied the exact asymptotic behaviour of solutions which blow up on the boundary for semilinear elliptic problems. 
In this paper, also applying Karamata regular variation theory, and constructing comparison functions, we show the existence and exact asymptotic behaviour of the unique solution near the boundary to problem (1.1).

First we recall a basic definition and a basic property to Karamata regular variation theory [13].

Definition 1.1. A positive measurable function $g$ defined on some neighborhood $(0, b)$ for some $b>0$, is called regularly varying at zero with index $\beta$, written $g \in \mathrm{RVZ}_{\beta}$ if for each $\xi>0$ and some $\beta \in \mathbb{R}$,

$$
\lim _{t \rightarrow 0^{+}} \frac{g(\xi t)}{g(t)}=\xi^{\beta}
$$

When $\beta=0$, we have the following definition.

Definition 1.2. A positive measurable function $L$ defined on some neighborhood $(0, b)$ for some $b>0$, is called slowly varying at zero, written $L \in \mathrm{RVZ}_{0}$ if for each $\xi>0$,

$$
\lim _{t \rightarrow 0^{+}} \frac{L(\xi t)}{L(t)}=1 .
$$

It follows by Definitions 1.1 and 1.2 that if $g \in \mathrm{RVZ}_{\beta}$, it can be represented in the form

$$
g(t)=t^{\beta} L(t)
$$

Lemma 1.3 (representation theorem). The function $L$ is slowly varying at zero if and only if it may be written in the form

$$
L(t)=c(t) \exp \left(\int_{t}^{b} \frac{y(s)}{s} d s\right), \quad 0<t<b,
$$

for some $b>0$, where $c(t)$ is a bounded measurable function, $y(t)$ is a continuous function on $[0, b]$, and for $t \rightarrow 0^{+}, y(t) \rightarrow 0$ and $c(t) \rightarrow C_{0}$, with $C_{0}>0$.

If $c(t)$ is replaced by its limit at zero $C_{0}$, a slowly varying function $L_{0} \in C^{1}(0, b]$ of the form

$$
L_{0}(t)=C_{0} \exp \left(\int_{t}^{b} \frac{y(s)}{s} d s\right), \quad 0<t<b
$$

where $y \in C[0, b]$ with $y(0)=0$, is obtained.

Such a function $L_{0}$ is called a normalised slowly varying at zero.

As an important subclass of $\mathrm{RVZ}_{\beta}$, it is defined as

$$
\mathrm{NRVZ}_{\beta}=\left\{g \in \mathrm{RVZ}_{\beta}: g(t) / t^{\beta} \text { is a normalised slowly varying at zero }\right\} .
$$

Our main results are as follows. 
Theorem 1.4. Let $k \in C^{\alpha}(\Omega)$ be nonnegative, $g$ satisfy $\left(g_{1}\right)$ and $g \in \mathrm{NRVZ}_{-\gamma}$ with $\gamma>1$. Suppose that there exists a nonnegative constant $c_{0}$ such that

(k) $\lim _{d(x) \rightarrow 0}(k(x) / g(\psi(d(x))))=c_{0}$;

then problem (1.1) has a unique solution $u \in C(\bar{\Omega}) \cap C^{2+\alpha}(\Omega)$ satisfying

$$
\lim _{d(x) \rightarrow 0} \frac{u(x)}{\psi(d(x))}=\xi_{0}
$$

where $\xi_{0}$ is the unique positive solution to the following equation:

$$
\xi^{-1-\gamma}=1+\frac{c_{0}}{\xi}
$$

and $\psi \in C[0, a] \cap C^{2}(0, a]$ is uniquely determined by

$$
\int_{0}^{\psi(t)} \frac{d s}{\sqrt{2 G(s)}}=t, \quad G(t)=\int_{t}^{a} g(s) d s, \quad a>0, t \in(0, a] .
$$

Moreover, $\psi \in \mathrm{NRVZ}_{2 /(1+\gamma)}$, and there exists $L_{0} \in \mathrm{NRVZ}_{0}$ such that

$$
\lim _{d(x) \rightarrow 0} \frac{u(x)}{L_{0}(d(x))(d(x))^{2 /(1+\gamma)}}=\xi_{0} .
$$

In particular, if $g(u)=u^{-\gamma}, \gamma>1$, then $\psi(s)=c s^{2 /(1+\gamma)}, c=\left[(1+\gamma)^{2} / 2(\gamma-1)\right]^{1 /(1+\gamma)}$, the unique solution $u$ to problem (1.1) satisfies

$$
\lim _{d(x) \rightarrow 0} \frac{u(x)}{[d(x)]^{2 /(1+\gamma)}}=\left[\frac{(1+\gamma)^{2}}{2(\gamma-1)}\right]^{1 /(1+\gamma)} \xi_{0} .
$$

Remark 1.5. In Section 2, we will see that $g \in \mathrm{NRVZ}_{-\gamma}$ with $\gamma>1 \operatorname{implies}_{\lim _{s \rightarrow 0^{+}}} g(s)=\infty$ and $G(t)<\infty, t>0$.

Remark 1.6. By the maximum principle [9], one easily sees that problem (1.1) has at most one solution in $C^{2}(\Omega) \cap C(\bar{\Omega})$.

Remark 1.7. Related to the above result, we raise the following open problem: when $k \leq 0$ on $\Omega$ and $c_{0}<0$, what is the exact asymptotic behaviour of the unique solution near the boundary to problem (1.1)?

The outline of this article is as follows. In Section 2, we continue to recall some basic properties to Karamata regular variation theory. In Section 3, we prove the asymptotic behaviour of the unique solution $u$ in Theorem 1.4. Finally we show existence of solutions to problem (1.1). 


\section{Some basic properties of Karamata regular variation theory}

Let us continue to recall some basic properties of Karamata regular variation theory (see [13]).

LEMMA 2.1. If $L$ is slowly varying at zero, then

(i) for every $\theta>0$ and $t \rightarrow 0^{+}$,

$$
t^{-\theta} L(t) \longrightarrow \infty, \quad t^{\theta} L(t) \longrightarrow 0
$$

(ii) for $a>0$ and $t \rightarrow 0^{+}$,

$$
\int_{t}^{a} s^{\beta} L(s) d s \cong(-\beta-1)^{-1} t^{1+\beta} L(t), \quad \text { for } \beta<-1 .
$$

Let $\Psi$ be nondecreasing on $\mathbb{R}$; define (as in [13]) the inverse of $\Psi$ by

$$
\Psi^{\leftarrow}(t)=\inf \{s: \Psi(s) \geq t\}
$$

Lemma 2.2 [13, Proposition 0.8]. The following hold:

(i) if $f_{1} \in \mathrm{RVZ}_{\rho_{1}}, f_{2} \in \mathrm{RVZ}_{\rho_{2}}$ with $\lim _{t \rightarrow 0^{+}} f_{2}(t)=0$, then $f_{1} \circ f_{2} \in \mathrm{RVZ}_{\rho_{1} \rho_{2}}$;

(ii) if $\Psi$ is nondecreasing on $(0, a), \lim _{t \rightarrow 0^{+}} \Psi(t)=0$, and $\Psi \in \mathrm{RVZ}_{\rho}$ with $\rho \neq 0$, then $\Psi^{\leftarrow} \in \mathrm{RVZ}_{\rho^{-1}}$.

By the above lemmas, we can directly obtain the following results.

Corollary 2.3. If $g$ satisfies $\left(\mathrm{g}_{1}\right)$ and $g \in \mathrm{NRVZ}_{-\gamma}$ with $\gamma>1$, then

$$
\begin{aligned}
& g(t)=t^{-\gamma} L_{0}(t), \quad \int_{0}^{1} g(t) d t=\infty, \\
& \lim _{t \rightarrow 0^{+}} \frac{G(t)}{g(t)}=0, \quad \lim _{t \rightarrow 0^{+}} \frac{\operatorname{tg}(t)}{G(t)}=\gamma-1,
\end{aligned}
$$

where $L_{0}$ is a normalised slowly varying function at zero.

Corollary 2.4. Under the assumptions in Theorem 1.4, $\psi \in \mathrm{NRVZ}_{2 /(1+\gamma)}$.

Proof. Let $f_{1}(t)=\int_{0}^{t}(d s / \sqrt{2 G(s)})$. By the l'Hospital rule and Corollary 2.3, we can easily see that

$$
\lim _{t \rightarrow 0^{+}} \frac{t f_{1}^{\prime}(t)}{f_{1}(t)}=1+\lim _{t \rightarrow 0^{+}} \frac{\operatorname{tg}(t)}{2 G(t)}=\frac{1+\gamma}{2} .
$$

It follows by Lemma 2.2 and [2] that $f_{1} \in \mathrm{NRVZ}_{(1+\gamma) / 2}$ and $\psi=f_{1}^{-1} \in \mathrm{NRVZ}_{2 /(1+\gamma)}$. 


\section{The exact asymptotic behaviour}

First we give some preliminary considerations.

Lemma 3.1. Let $g, k$, and $\psi$ be in Theorem 1.4. The following hold:

(i) $\lim _{t \rightarrow 0^{+}} \psi^{\prime}(t)=\psi^{\prime}(0)=+\infty$;

(ii) $\lim _{t \rightarrow 0^{+}}(\sqrt{2 G(\psi(t))} / g(\psi(t)))=0$.

Proof. By (1.14), we see by a direct calculation that

$$
\psi^{\prime}(t)=\sqrt{2 G(\psi(t))}, \quad-\psi^{\prime \prime}(t)=g(\psi(t)), \quad 0<t<a .
$$

(i) By Corollary 2.4, Lemma 2.1 and $\gamma>1$, we see that there exists $L_{0} \in \mathrm{NRVZ}_{0}$ such that

$$
\psi(t)=t^{2 /(\gamma+1)} L_{0}(t), \quad \psi^{\prime}(t)=t^{(1-\gamma) /(\gamma+1)} L_{0}(t)\left(\frac{2}{\gamma+1}-y(t)\right) .
$$

So $\lim _{t \rightarrow 0^{+}} \psi^{\prime}(t)=+\infty$.

(ii) By $\left(g_{1}\right)$ and Corollary 2.3, we see that

$$
\lim _{t \rightarrow 0^{+}} \frac{\sqrt{2 G(\psi(t))}}{g(\psi(t))}=\lim _{u \rightarrow 0^{+}} \frac{\sqrt{2 G(u)}}{g(u)}=\lim _{u \rightarrow 0^{+}}\left(\frac{2 G(u)}{g(u)}\right)^{1 / 2} \lim _{u \rightarrow 0^{+}}\left(\frac{1}{g(u)}\right)^{1 / 2}=0 .
$$

The exact asymptotic behaviour. Let $\xi_{0}$ be the unique positive solution to problem (1.13). For $\varepsilon \in\left(0, \xi_{0}^{-1-\gamma} / 4\right)$, denote

$$
a_{0}=\xi_{0}^{-1-\gamma}=1+\frac{c_{0}}{\xi_{0}}, \quad \xi_{1 \varepsilon}^{-1-\gamma}=a_{0}-2 \varepsilon, \quad \xi_{2 \varepsilon}^{-1-\gamma}=a_{0}+2 \varepsilon .
$$

Obviously, $a_{0} \geq 1, c_{0} / a_{0} \xi_{0}=\left(a_{0}-1\right) / a_{0} \in[0,1)$, and $\xi_{0} / 2<\xi_{2 \varepsilon}<\xi_{0}<\xi_{1 \varepsilon}<2 \xi_{0}$. Moreover, it follows by Taylor's formula that

$$
c_{0}\left|\left(\frac{1}{\xi_{0}}-\frac{1}{\xi_{i \varepsilon}}\right)\right|=\frac{2 c_{0} \varepsilon}{a_{0} \xi_{0}(1+\gamma)}+o(\varepsilon)=\frac{2 \varepsilon\left(a_{0}-1\right)}{a_{0}(1+\gamma)}+o(\varepsilon), \quad i=1,2 .
$$

Thus there exist $\varepsilon_{1}>0$ and $\rho_{0} \in\left(2\left(a_{0}-1\right) / a_{0}(1+\gamma), 1\right)$ such that

$$
c_{0}\left|\left(\frac{1}{\xi_{0}}-\frac{1}{\xi_{i \varepsilon}}\right)\right|<\rho_{0} \varepsilon \quad \text { for } \varepsilon \in\left(0, \varepsilon_{1}\right) .
$$

For $\delta>0$, we define $\Omega_{\delta}=\{x \in \Omega: d(x) \leq \delta\}$. By the regularity of $\partial \Omega$ and Lemma 3.1, we can choose $\delta$ sufficiently small such that

(i) $d(x) \in C^{2}\left(\Omega_{\delta}\right)$;

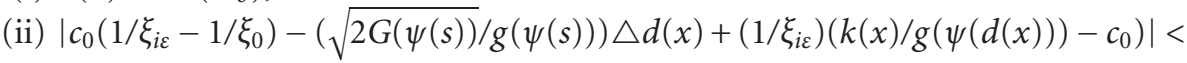
$\varepsilon$, for all $(s, x) \in(0, \delta) \times \Omega_{\delta}, i=1,2$;

(iii) $\left(\xi_{2 \varepsilon} g(\psi(d(x))) / g\left(\xi_{2 \varepsilon} \psi(d(x))\right)\right)\left(\xi_{2 \varepsilon}^{-1-\gamma}-\varepsilon\right)<1<\left(\xi_{1 \varepsilon} g(\psi(d(x))) / g\left(\xi_{1 \varepsilon} \psi(d(x))\right)\right)$ $\left(\xi_{1 \varepsilon}^{-1-\gamma}+\varepsilon\right)$ in $\Omega_{\delta}$. 
For any $x \in \Omega_{\delta}$, define $\bar{u}=\xi_{1 \varepsilon} \psi(d(x))$, and $\underline{u}=\xi_{2 \varepsilon} \psi(d(x))$. It follows by $|\nabla d(x)|=1$ that

$$
\begin{aligned}
& \triangle \bar{u}(x)+g(\bar{u}(x))-k(x) \\
& =g\left(\xi_{1 \varepsilon} \psi(d(x))\right)+\xi_{1 \varepsilon} \psi^{\prime \prime}(d(x))+\xi_{1 \varepsilon} \psi^{\prime}(d(x)) \triangle d(x)-k(x) \\
& =\xi_{1 \varepsilon} g(\psi(d(x)))\left[\frac{g\left(\xi_{1 \varepsilon} \psi(d(x))\right)}{\xi_{1 \varepsilon} g(\psi(d(x)))}-\left(1+\frac{c_{0}}{\xi_{0}}\right)-c_{0}\left(\frac{1}{\xi_{1 \varepsilon}}-\frac{1}{\xi_{0}}\right)\right. \\
& \left.+\frac{\sqrt{2 G(\psi(d(x)))}}{g(\psi(d(x)))} \Delta d(x)-\frac{1}{\xi_{1 \varepsilon}}\left(\frac{k(x)}{g(\psi(d(x)))}-c_{0}\right)\right] \\
& \leq \xi_{1 \varepsilon} g(\psi(d(x)))\left[\left(1+\frac{\lambda c_{0}}{\xi_{0}}-\varepsilon\right)-\left(1+\frac{c_{0}}{\xi_{0}}\right)-c_{0}\left(\frac{1}{\xi_{1 \varepsilon}}-\frac{1}{\xi_{0}}\right)\right. \\
& \left.+\frac{\sqrt{2 G(\psi(d(x)))}}{g(\psi(d(x)))} \Delta d(x)-\frac{1}{\xi_{1 \varepsilon}}\left(\frac{k(x)}{g(\psi(d(x)))}-c_{0}\right)\right] \leq 0 ; \\
& \triangle \underline{u}(x)+g(\underline{u}(x))-k(x) \\
& =g\left(\xi_{2 \varepsilon} \psi(d(x))\right)+\xi_{2 \varepsilon} \psi^{\prime \prime}(d(x))+\xi_{2 \varepsilon} \psi^{\prime}(d(x)) \triangle d(x)-k(x) \\
& =\xi_{2 \varepsilon} g(\psi(d(x)))\left[\frac{g\left(\xi_{2 \varepsilon} \psi(d(x))\right)}{\xi_{2 \varepsilon} g(\psi(d(x)))}-\left(1+\frac{c_{0}}{\xi_{0}}\right)-c_{0}\left(\frac{1}{\xi_{2 \varepsilon}}-\frac{1}{\xi_{0}}\right)\right. \\
& \left.+\frac{\sqrt{2 G(\psi(d(x)))}}{g(\psi(d(x)))} \Delta d(x)-\frac{1}{\xi_{2 \varepsilon}}\left(\frac{k(x)}{g(\psi(d(x)))}-c_{0}\right)\right] \\
& \geq \xi_{2 \varepsilon} g(\psi(d(x)))\left[\left(1+\frac{c_{0}}{\xi_{0}}+\varepsilon\right)-\left(1+\frac{c_{0}}{\xi_{0}}\right)-c_{0}\left(\frac{1}{\xi_{2 \varepsilon}}-\frac{1}{\xi_{0}}\right)\right. \\
& \left.+\frac{\sqrt{2 G(\psi(d(x)))}}{g(\psi(d(x)))} \Delta d(x)-\frac{1}{\xi_{2 \varepsilon}}\left(\frac{k(x)}{g(\psi(d(x)))}-c_{0}\right)\right] \geq 0 .
\end{aligned}
$$

Let $u \in C(\bar{\Omega}) \cap C^{2+\alpha}(\Omega)$ be the unique solution to problem (1.1). We assert

$$
\xi_{2 \varepsilon} \psi(d(x))=\underline{u}(x) \leq u(x) \leq \bar{u}(x)=\xi_{1 \varepsilon} \psi(d(x)) \quad \forall x \in \Omega_{\delta} .
$$

In fact, denote $\Omega_{\delta}=\Omega_{\delta_{+}} \cup \Omega_{\delta_{-}}$, where $\Omega_{\delta_{+}}=\left\{x \in \Omega_{\delta}: u(x) \geq \underline{u}(x)\right\}$ and $\Omega_{\delta_{-}}=\{x \in$ $\left.\Omega_{\delta}: u(x)<\underline{u}(x)\right\}$. We see by $\left(\mathrm{g}_{1}\right)$ that

$$
-\Delta(u-\underline{u})(x) \geq g(u(x))-g(\underline{u}(x))>0, \quad x \in \Omega_{\delta_{-}} .
$$

Since $(u-\underline{u})(x)=0, x \in \partial \Omega_{\delta_{-}}$, we see by the maximum principle [9, Theorem 2.3] that $u(x) \geq \underline{u}(x), x \in \Omega_{\delta_{-}}$, that is, $\Omega_{\delta_{-}}=\varnothing$. Thus $\xi_{2 \varepsilon} \psi(d(x)) \leq u(x)$, for all $x \in \Omega_{\delta}$. In the same way, we can see that $u(x) \leq \xi_{1 \varepsilon} \psi(d(x))$, for all $x \in \Omega_{\delta}$. Let $\varepsilon \rightarrow 0$, we see that $\lim _{d(x) \rightarrow 0}(u(x) / \psi(d(x)))=\xi_{0}$. By Corollary 2.4, the proof is finished. 


\section{Existence of solutions}

First we introduce a sub-supersolution method with the boundary restriction (see [5]).

We consider the more general following problem:

$$
-\Delta u=f(x, u), \quad u>0, x \in \Omega,\left.u\right|_{\partial \Omega}=0 .
$$

Definition 4.1. A function $\underline{u} \in C^{2+\alpha}(\Omega) \cap C(\bar{\Omega})$ is called a subsolution to problem (4.1) if

$$
-\Delta \underline{u} \leq f(x, \underline{u}), \quad \underline{u}>0, x \in \Omega,\left.\underline{u}\right|_{\partial \Omega}=0 .
$$

Definition 4.2. A function $\bar{u} \in C^{2+\alpha}(\Omega) \cap C(\bar{\Omega})$ is called a supersolution to problem (4.1) if

$$
-\Delta \bar{u} \geq f(x, \bar{u}), \quad \bar{u}>0, x \in \Omega,\left.\bar{u}\right|_{\partial \Omega}=0 .
$$

Lemma 4.3 [5, Lemma 3]. Let $f(x, s)$ be locally Hölder continuous in $\Omega \times(0, \infty)$ and continuously differentiable with respect to the variable s. Suppose problem (4.1) has a supersolution $\bar{u}$ and a subsolution $\underline{u}$ such that $\underline{u} \leq \bar{u}$ on $\Omega$, then problem (4.1) has at least one solution $u \in C^{2+\alpha}(\Omega) \cap C(\bar{\Omega})$ in the ordered interval $[\underline{u}, \bar{u}]$.

Denote

$$
|u|_{\infty}=\max _{x \in \bar{\Omega}}|u(x)|, \quad u \in C(\bar{\Omega})
$$

Now we apply Lemma 4.3 to consider existence of solutions to problem (1.1).

Let $u_{0} \in C^{2+\alpha}(\Omega) \cap C(\bar{\Omega})$ be the unique solution to problem (1.2). Obviously, $\bar{u}=u_{0}$ is a supersolution to problem (1.1). To construct a subsolution to problem (1.1), let $w \in$ $C^{2+\alpha}(\Omega) \cap C^{1}(\bar{\Omega})$ be the unique solution to the following problem:

$$
-\Delta w=1, \quad w>0, \quad x \in \Omega,\left.\quad w\right|_{\partial \Omega}=0 .
$$

It follows by the Höpf maximum principle that there exist positive constants $c_{1}$ and $c_{2}$ such that

$$
c_{1} d(x) \leq w(x) \leq c_{2} d(x) \quad \forall x \in \Omega, \quad \nabla w(x) \neq 0 \quad \forall x \in \partial \Omega .
$$

Let $a>|w|_{\infty}$ in (1.14) and

$$
M_{0}=\sup _{x \in \bar{\Omega}}\left(|\nabla w(x)|^{2}+\frac{\sqrt{2 G(\psi(w(x)))}}{g(\psi(w(x)))}\right), \quad M_{1}=\sup _{x \in \bar{\Omega}}\left(\frac{k(x)}{g(\psi(d(x)))} \frac{g\left(\psi\left(c_{2}^{-1} w(x)\right)\right)}{g(\psi(w(x)))}\right) .
$$

By Corollary 2.4 and Lemma 2.2, we see that $g \circ \psi \in \mathrm{NRVZ}_{-2 \gamma /(1+\gamma)}$. It follows by the assumption (k) and Lemma 3.1 that $M_{0}, M_{1} \in(0, \infty)$. 
Define

$$
\underline{u}=m \psi(w(x))
$$

where $m$ is a positive constant to be chosen.

It follows that

$$
\begin{aligned}
& -\Delta \underline{u}(x)+k(x) \\
& \quad=g(\psi(w(x)))\left[m\left(|\nabla w(x)|^{2}+\frac{\sqrt{2 G(\psi(w(x)))}}{g(\psi(w(x)))}\right)+\frac{k(x)}{g(\psi(d(x)))} \frac{g(\psi(d(x)))}{g(\psi(w(x)))}\right] \\
& \quad \leq\left(m M_{0}+M_{1}\right) g(\psi(w(x))), \quad x \in \Omega .
\end{aligned}
$$

Let us analyze the function

$$
F_{m}(x)=\frac{g(m \psi(w(x)))}{g(\psi(w(x)))}, \quad x \in \Omega .
$$

By $\lim _{x \rightarrow \partial \Omega} F_{m}(x)=m^{-\gamma}$, we see that there exist positive constants $\delta_{0}$ and $m_{0}$ such that for $m \in\left(0, m_{0}\right)$,

$$
F_{m}(x) \geq m M_{0}+M_{1} \quad \forall x \in \bar{\Omega}_{\delta_{0}},
$$

where $\Omega_{\delta_{0}}=\left\{x \in \Omega: d(x)<\delta_{0}\right\}$ and $m_{0}$ is the unique positive root of the equation

$$
m^{-\gamma}=2\left(m M_{0}+M_{1}\right)
$$

Let

$$
A_{0}=\max _{x \in \bar{\Omega} / \Omega_{\delta_{0}}} \psi(w(x)), \quad a_{0}=\min _{x \in \bar{\Omega} / \Omega_{\delta_{0}}} \psi(w(x))
$$

It follows by $\left(\mathrm{g}_{1}\right)$ that there exists $m_{1}>0$ such that

$$
F_{m}(x) \geq \frac{g\left(m A_{0}\right)}{g\left(a_{0}\right)} \geq m M_{0}+M_{1} \quad \forall m \in\left(0, m_{1}\right) .
$$

Thus $-\Delta \underline{u}(x) \leq g(\underline{u}(x))-k(x), x \in \Omega$, that is, $\underline{u}=m \psi(w(x))$ is a subsolution to problem (1.1) for $0<m<\min \left\{m_{1}, m_{0}\right\}$. Moreover, we see by the maximum principle [9, Theorem 2.3] that $\underline{u} \leq u_{0}$ on $\bar{\Omega}$ and by Lemma 4.3 that problem (1.1) has at least one solution $u \in C^{2+\alpha}(\Omega) \cap C(\bar{\Omega})$ in ordered interval $\left[\underline{u}, u_{0}\right]$.

The proof is complete.

\section{Acknowledgment}

This work is supported in part by the National Natural Science Foundation of China under Grant no. 10071066. 


\section{References}

[1] C. Aranda and T. Godoy, On a nonlinear Dirichlet problem with a singularity along the boundary, Differential and Integral Equations 15 (2002), no. 11, 1313-1324.

[2] F.-C. Cîrstea and Y. Du, General uniqueness results and variation speed for blow-up solutions of elliptic equations, Proceedings of the London Mathematical Society. Third Series 91 (2005), no. 2, 459-482.

[3] F.-C. Cîrstea and V. D. Rădulescu, Asymptotics for the blow-up boundary solution of the logistic equation with absorption, Comptes Rendus Mathématique. Académie des Sciences. Paris 336 (2003), no. 3, 231-236.

[4] M. G. Crandall, P. H. Rabinowitz, and L. Tartar, On a Dirichlet problem with a singular nonlinearity, Communications in Partial Differential Equations 2 (1977), no. 2, 193-222.

[5] S. Cui, Existence and nonexistence of positive solutions for singular semilinear elliptic boundary value problems, Nonlinear Analysis. Theory, Methods \& Applications. Series A: Theory and Methods 41 (2000), no. 1-2, 149-176.

[6] G. Díaz and R. Letelier, Explosive solutions of quasilinear elliptic equations: existence and uniqueness, Nonlinear Analysis. Theory, Methods \& Applications. Series A: Theory and Methods 20 (1993), no. 2, 97-125.

[7] W. Fulks and J. S. Maybee, A singular non-linear equation, Osaka Journal of Mathematics 12 (1960), 1-19.

[8] M. Ghergu and V. D. Rădulescu, Bifurcation and asymptotics for the Lane-Emden-Fowler equation, Comptes Rendus Mathématique. Académie des Sciences. Paris 337 (2003), no. 4, 259-264.

[9] D. Gilbarg and N. S. Trudinger, Elliptic Partial Differential Equations of Second Order, 3rd ed., Springer, Berlin, 1998.

[10] J.-M. Lasry and P.-L. Lions, Nonlinear elliptic equations with singular boundary conditions and stochastic control with state constraints. I. The model problem, Mathematische Annalen 283 (1989), no. 4, 583-630.

[11] A. C. Lazer and P. J. McKenna, On a singular nonlinear elliptic boundary-value problem, Proceedings of the American Mathematical Society 111 (1991), no. 3, 721-730.

[12] A. Nachman and A. Callegari, A nonlinear singular boundary value problem in the theory of pseudoplastic fluids, SIAM Journal on Applied Mathematics 38 (1980), no. 2, 275-281.

[13] S. I. Resnick, Extreme Values, Regular Variation, and Point Processes, Applied Probability. A Series of the Applied Probability Trust, vol. 4, Springer, New York, 1987.

[14] C. A. Stuart, Existence and approximation of solutions of non-linear elliptic equations, Mathematische Zeitschrift 147 (1976), no. 1, 53-63.

[15] Z. Zhang, The asymptotic behaviour of the unique solution for the singular Lane-Emden-Fowler equation, Journal of Mathematical Analysis and Applications 312 (2005), no. 1, 33-43.

[16] Z. Zhang and J. Cheng, Existence and optimal estimates of solutions for singular nonlinear Dirichlet problems, Nonlinear Analysis. Theory, Methods \& Applications. Series A: Theory and Methods 57 (2004), no. 3, 473-484.

[17] Z. Zhang and J. Yu, On a singular nonlinear Dirichlet problem with a convection term, SIAM Journal on Mathematical Analysis 32 (2000), no. 4, 916-927.

Zhijun Zhang: Department of Mathematics and Informational Science, Yantai University, Yantai, Shandong 264005, China

E-mail address: zhangzj@ytu.edu.cn

Jianning Yu: College of Mathematics, Physics and Software Engineering, Lanzhou Jiaotong

University, Lanzhou, Gansu 730070, China

E-mail address: yujn@mail.lzjtu.cn 